\title{
Effect of parameters on the morphology and fibre diameters of edible electrospun chitosan-cellulose acetate-gelatin hybrid nanofibres
}

\author{
Jaruayporn Somsap ${ }^{1, *}$, Kobsak Kanjanapongkul ${ }^{2}$, and Racha Tepsorn ${ }^{1}$ \\ ${ }^{1}$ Department of Food Science and Technology, Faculty of Science and Technology, Thammasasat University (Rangsit Center), Phatum \\ Thani, Thailand \\ ${ }^{2}$ Department of Food Engineering, Faculty of Engineering at Kamphaeng Saen, Kasetsart University, Kamphaeng Saen Campus, \\ Nakhon Pathom, Thailand
}

\begin{abstract}
Electrospinning is the favorite process to fabricate fibres with diameter in the range nanoscale through the action of electric field. In this study, 3-7\% chitosan, $18.0 \%$ cellulose acetate and $30.0 \%$ gelatin solution in aqueous $80 \%$ acetic acid solution were blended at the volume ratio of $4: 1: 5$ have been successfully electrospun. The effect of processing parameters and the concentration of the polymer solution on the morphology and diameter of electrospun were investigated. The morphology and diameter of electrospun fibres were observed by scanning electron microscope. The diameters of chitosan-cellulose acetate-gelatin nanofibres ranging from 78.94 to $421.05 \mathrm{~nm}$. The results showed that the fibre diameters increased when the solution concentration and flow rate were increased, whereas the fibre diameters decreased when the applied voltage and distance between tip to collector were increased. The conditions of the solution concentration $18.8 \% \mathrm{wt}$, applied voltage at $23 \mathrm{kV}$, flow rate at $11.67 \mu \mathrm{L} / \mathrm{min}$ and collector distance at $10 \mathrm{~cm}$ were selected to prepares the desirable electrospun nanofibres for applications and the further research.
\end{abstract}

Keyword: Electrospinning, electrospun nanofibre, edible electrospun

\section{Introduction}

Nanofibres was a nanomaterial that was tiny fibre with diameter in the range of 1-100 nanometre. It has a variety technique to prepared nanofibres such as drawing, template synthesis, phase separation, selfassembly and electrospinning [1-3]. However, electrospinning was the most attractive process to fabricate nanofibres due to that the apparatus was uncomplicate and flexible for the raw material selection. The basis apparatus of electrospinning consists of (i) high voltage power supply, (ii) syringe with a blunt ended stainless needle, (iii) syringe pump and (iv) ground collector $[4,5]$. In the electrospinning process, polymer solution is taken in the syringe with needle then the positive electrode which has a high voltage power supply was connected to a needle while the negative electrode was connected to a ground collector. When the high voltage was applied, the droplet shape at the end of needle will be changed from a semicircle into a conical shape as known "Taylor's cone" due to the charge charge repulsion [5-8]. During the jet transportation cross the high voltage electric field, the whipping instability motion and stretching of fibre will be observed. The solvent will be evaporated. Thin and dry fibres on the collector are obtained [8,9]. These fibres were called "electrospun nanofibres".

Electrospun nanofibres presented the outstanding properties such as high surface area to volume or mass ratio, light weight, small inter-fibrous pore size with high porosity [5,7,10]. They were used for several applications such as filtration membranes, nanofiber composites, wound dressing, tissue scaffolding, drug delivery systems and food packaging [7,11]. Morphology and fibre diameters of electrospun depended on various parameters that including solution properties, process parameters, and ambient parameters $[2,5,12]$. Understanding the parameters will help to fabricate the desirable electrospun nanofibers.

Chitosan, cellulose acetate and gelatin are the natural polymers that were selected in this study due to there are edible, biocompatible, biodegradable, eco-friendly and low toxicity. Dhandayuthapani et al. [1] and Haider et al. [13] successfully fabricated electrospun nanofibre mats from chitosan-gelatin blend for applications in the biomedical field. In addition, several reports demonstrated that cellulose acetate -gelatin blend could use for electrospun nanofibre mats preparation as well $[14,15]$. However, as far as we have known that the availability data for the electrospun nanofibre from the blend of chitosan-cellulose acetate-gelatin hybrid was

\footnotetext{
Corresponding author: jaruayporn12@gmail.com
} 
never presented. The aim of this study was to investigate the effect parameter including of concentration of polymer solution, applied voltage, flow rate and distance between tip to collector on the electrospinning process. The morphologies of electrospun including diameters and diameter distribution were determined. The outcomes of this study may give the optimum condition for fabricate uniform electrospun fibres with desirable properties for applications in food sector.

\section{Materials and methods}

Chitosan powder (degree of deacetylation 95.04\%) and gelatin powder ( 250 bloom) were purchased from Thai Food and Chemical Co.Ltd (Thailand). Cellulose Acetate (CA, average $\mathrm{Mn} \sim 30,000$ ) was purchased from Aldrich Co. Ltd ( USA) . Glacial acetic acid ( $>99 \%$ ) was purchased from QRec (New Zealand). Chitosan, CA and gelatin were separately dissolved in $80 \%$ acetic acid (v/v) under continuous stirring at room temperature until completely dissolved. Then, the Chitosan-GelatinCellulose acetate were prepared at the volume ratio of $4: 1: 5$, respectively. The concentration of Chitosan-CAGelatin in mixed polymer were $18.0,18.8$ and $19.6 \%$ wt with the constant concentration of Gelatin and CA. The morphology and diameters of the electrospun nanofibres was investigated using scanning electron microscopy ( SEM JSM-7800F, JEOL, MA, USA) . Statistical analyses were performed by ANOVA with 3 replicates. Differentiate analysis among the data was analyzed using Duncan at $p$-value $<0.05$.

\section{Electrospinning set up and process}

The mixed Chitosan-CA-Gelatin solution was loaded in $10 \mathrm{~mL}$ plastic syringe connected with 18 gauges metal needle. The flow rate $(11.67-25.00 \mu \mathrm{L} / \mathrm{min}$.) was controlled by a syringe pump (New Era NE-300, USA). A high voltage DC power supply $(20-26 \mathrm{kV})$ was applied at distance of $8-12 \mathrm{~cm}$. The electrospinning was performed at room temperature $\left(27 \pm 2^{\circ} \mathrm{C}\right)$ and all electrospun nanofibres were dried overnight to remove residual solvent.

\section{Results and discussion}

\section{Effect of solution concentration}

The solution of Chitosan in acetic acid was varied from $8.0 \%, 9.0 \%, 10.0 \%, 11.0 \%$ and $12.0 \%$ wt at $25 \mathrm{kV}$, the distance collector was $8 \mathrm{~cm}$ and flow rate was 25.00 $\mu \mathrm{L} / \mathrm{min}$. It was found that no fibre formation and only droplets were collected as shown in Fig. 1. Average droplet's size was $1.042-43.235 \mu \mathrm{m}$. The diameter and roughness were increased by increasing Chitosan concentration. Gelatin electrospun fibre mats were obtained by the $30.0 \%$ wt gelatin at $23 \mathrm{kV}$, flow rate was $11.67 \mu \mathrm{L} / \mathrm{min}$. The electrospun fibre mats were only obtained by $18.0 \%$ wt $\mathrm{CA}$ at $25 \mathrm{kV}$, flow rate was 50.00 $\mu \mathrm{L} / \mathrm{min}$. and the distance was $10 \mathrm{~cm}$ (Fig. $2 \mathrm{~A}$ and $2 \mathrm{~B}$ ). The diameter of Gelatin fibres ranged from 384.61$2564.10 \mathrm{~nm}$ and average diameter was $1061.53 \pm 500.25$ $\mathrm{nm}$. On the other hand, Oraby et al. [16] reported that no fibres at $30.0 \% \mathrm{wt}$ Gelatin concentration was investigated, the fibres were first appeared at $40.0 \% \mathrm{wt}$ Gelatin (69-138 nm) and increased to when Gelatin concentration was increased.

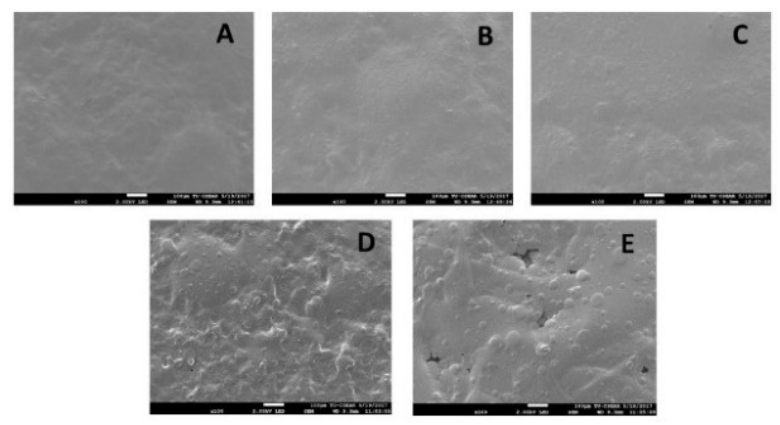

Fig 1. SEM images (100x) of electrospun drops at different Chitosan concentration (A) $8.0 \%$, (B) $9.0 \%$, (C) $10.0 \%$, (D) $11.0 \%$ and (E) $12.0 \% \mathrm{wt}$

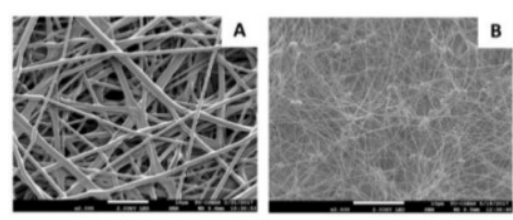

Fig. 2. SEM images of electrospun fibre mats $(A)$ Gelatin electrospun; (B) CA electrospun (2,000x (A) and 2,500x (B))

Whereas Haider et al. [13] showed that at below $15.0 \%$ wt Gelatin solution, the fibres were not detected and the smooth fibres electrospun nanofibres was firstly detected at $15.0 \%$ wt with average diameter of $149 \mathrm{~nm}$ at $20 \mathrm{kV}$ and the electrical field distance at $10 \mathrm{~cm}$. The increasing of the solution concentration could be resulted to the viscosity properties, especially the entanglement properties of polymer. At the high concentration, above entanglement concentration, the nanoscaled fibres would be no optained. However, the flat fibres and the ribbontype fibre were achived [12,17]. For the diameter of CA fibres ranged from 47.61-142.85 $\mathrm{nm}$ and the average diameter was $85.142 \pm 24.05 \mathrm{~nm}$. Moreover, beads were observed on the fibres. On the contrary, Han et al. [18] have reported that $\mathrm{CA}$ at higher concentrations than 17.0 $\mathrm{wt} \%$ in acetic acid could be electrospuned, long uniform nanofibres without beads were observed.

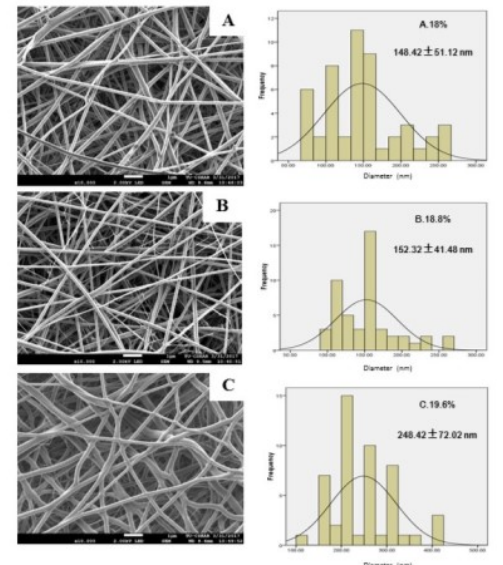

Fig. 3 SEM images $(10,000 x)$ of electrospun nanofibres and diameters distribution for the various solution (A) $18.0 \mathrm{wt} \%$ (B) $18.8 \mathrm{wt} \%$ and (C) $19.6 \mathrm{wt} \%$ 
Fig. 3 showed the Chitosan-CA-Gelatin nanofibres and diameter distribution obtained at concentration of 18.0, 18.8 and $19.6 \%$ wt in $80 \%$ acetic acid. All of solution concentrations of Chitosan-CA-Gelatin could be electrospuned. The diameter of Chitosan-CA-Gelatin nanofibres ranged from 78.94-421.05 $\mathrm{nm}$ and the average diameter were $148.42 \pm 51.12,152.32 \pm 41.48$ and $248.42 \pm 72.02 \mathrm{~nm}$, respectively. The fibres diameter were increased continuously by increasing polymer concentration from $18 \mathrm{wt} \%$ to $19.6 \mathrm{wt} \%$ (Fig. 4).

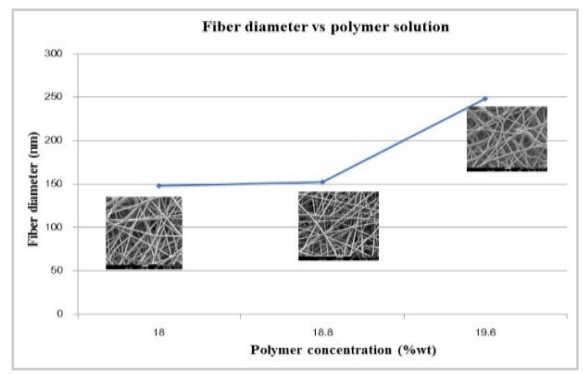

Fig. 4 The relation between the fibre diameters and polymer concentration

However, the diameters at 18.0 and $18.8 \mathrm{wt} \%$ solution concentration were different without significant $(p>0.05)$. The smooth, non-bead and continuously fibres were obtained. At $19.6 \mathrm{wt} \%$ solution concentration, the junction and sticky were observed due to the higher viscosity and surface tension of polymer jets [19]. In contrast, the diameter fibres were significant increased $(p<0.05)$. Polymer concentration and viscosity are related factor, when one increased, the other would be increased that resulted to the size of diameter fibres. At low concentration, polymeric micro or nano-particle will be obtained, the suitable concentration, smooth, uniform and continuous nanofibres was presented $[5,10,12,16]$.

\section{Effect of applied voltage}

Voltage involved the force in the electrical field. Applied voltage resulted on the formation of Taylor cone according to the repulsive force in an opposite direction of the surface tension. The overcome state resulted on the initiation of fine jet from the cone and flying to collector [5,6,12]. In this experiment, effect of applied voltage on fibre morphology at 20, 23 and $26 \mathrm{kV}$ were investigated. The average fibres diameters at 20,23 and $26 \mathrm{kV}$ were $170.21 \pm 42.15, \quad 152.32 \pm 41.48$ and $106.95 \pm 35.39 \mathrm{~nm}$ respectively. Smooth, non-beads and continuous fibres were obtained from all treatments. In addition, fibres diameters were decreased while applied voltage was increased (Fig.5 and 6), it was in accordance with the previous reports $[12,18]$. However, the beads of PVA/alginate was also detected [7]. Moreover, the electrospun fibres with bigger beads were obtained at 45 $\mathrm{kV}$. It was investigated that when applied voltage was increased, number of junctions and fibres diameters decreased while the size of beads increased. Effect of increasing voltage could be that was increased the density of charge in polymer solution, then the Taylor cone was formed, stretched and formation of smaller fibres due to the higher repulsive forces. Moreover, the more increasing of voltage, the more electric field strengths between needle and ground collector also increased. The polymer jet could fly to collector in short time that results in increasing of bead formation and/or larger fiber diameters $[5,7,12,19]$.
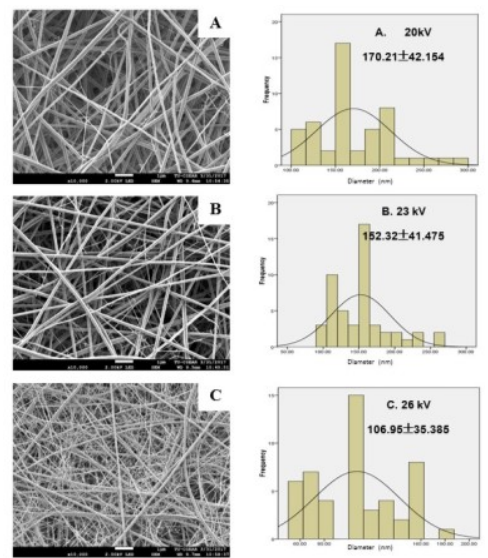

Fig. 5 SEM images $(10,000 x)$ of electrospun nanofibres and diameters distribution at different applied voltage (A) $20 \mathrm{kV}$ (B) $23 \mathrm{kV}$ and $(\mathrm{C}) 26 \mathrm{kV}$

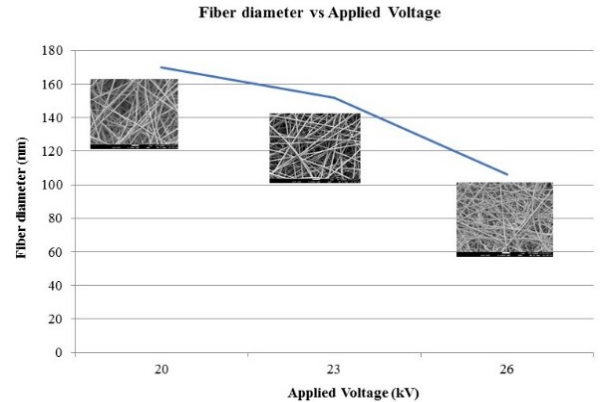

Fig.6 The relation between the fibre diameters and applied voltage

\section{Effect of flow rate}

The average fibres diameter at flow rates of 11.67 $\mu \mathrm{L} / \mathrm{min}, 16.67 \mu \mathrm{L} / \mathrm{min}$, and $25.00 \mu \mathrm{L} / \mathrm{min}$ were $152.32 \pm 41.48, \quad 154.21 \pm 45.40$ and $165.68 \pm 54.75 \mathrm{~nm}$ respectively. As show as Fig. 7 and 8. Smooth, non-bead and continuous fibres were obtained.

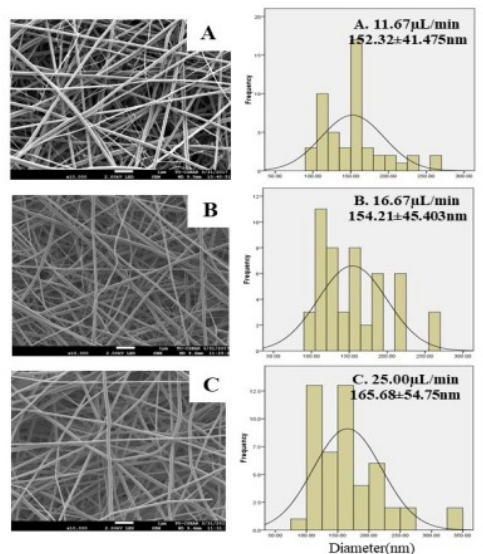

Fig. 7 SEM images $(10,000 x)$ of electrospun nanofibers and diameter distribution at the different flow rate;(A) $11.67 \mu \mathrm{L} / \mathrm{min}$ (B) $16.67 \mu \mathrm{L} / \mathrm{min}$ and (C) $25.00 \mu \mathrm{L} / \mathrm{min}$

The flow rate influence to the initiate droplet shape. At high flow rates, bigger droplet was formed, and the flying ability across electrical field was decreased. The 
thick fibres were formed on the collector. Therefore, the lower flow rate was more recommend $[8,12,21]$.

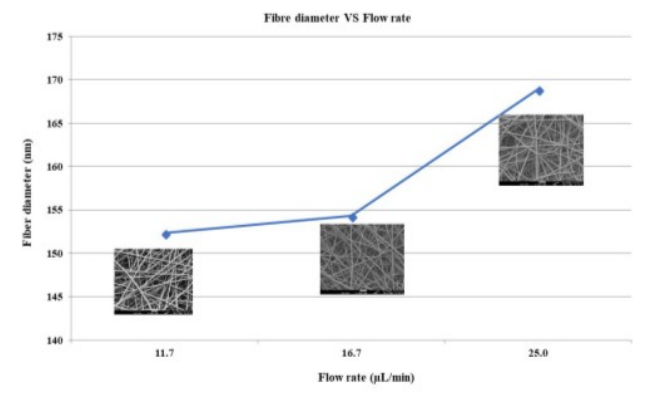

Fig. 8 Relation between the fibres diameters and flow rate

In this research, diameters at flow rates $11.67 \mu \mathrm{L} / \mathrm{min}$, and $16.67 \mu \mathrm{L} / \mathrm{min}$ were different without significant $(p>0.05)$.

\section{Effect of distance}

The distance showed a direct effect on the jet flying time and solvent evaporation time. At the short distance, bead formation, thick and wet fibres could be obtained, whereas at the longer, small bead, smooth and continuous fibres with fibres diameters decreasing could be obtained owing to the flying time of jet and completely evaporated $[2,12,19]$. The average diameter of electrospun Chitosan-CA-Gelatin fibres at distance 8 $\mathrm{cm}, 10 \mathrm{~cm}$, and $12 \mathrm{~cm}$ were $158.32 \pm 48.84,152.32 \pm 41.48$ and $146.74 \pm 40.22 \mathrm{~nm}$ respectively.

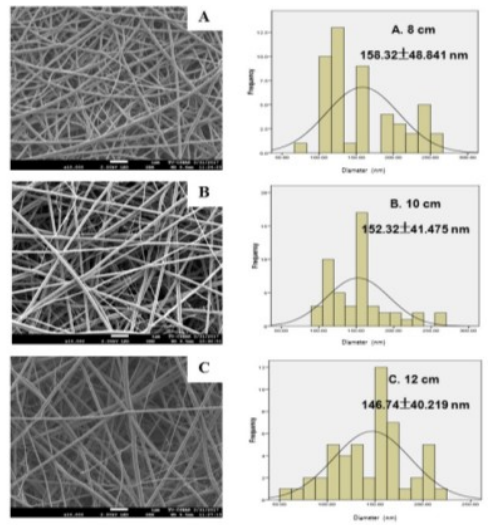

Fig.9 SEM images $(10,000 x)$ of electrospun nanofibers and diameter distribution at the different the collector distance (A) $8 \mathrm{~cm}$ (B) $10 \mathrm{~cm}$ and (C) $12 \mathrm{~cm}$

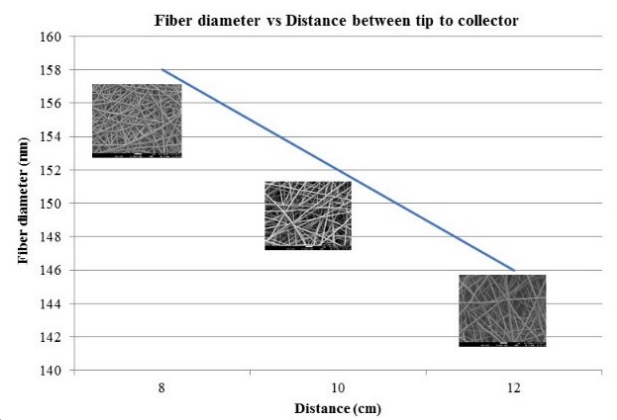

Fig. 10 Relation between the fibre diameters and collector distance

It could be indicated that fibres diameters decreases when distance was increased (Fig. 9 and 10). Increasing the distance could give time for the fluid jet to stretch and for the solvent to evaporate completely $[19,20]$.

\section{Conclusion}

Formation of Chitosan-CA-Gelatin was successfully obtained from the mixed of those at the ratio of $4: 1: 5$. According to the study, it can be indicated that the morphology of Chitosan-CA-Gelatin was depended on polymer concentration, applied voltage, polymer's flow rate and the distance between tip to collector. The optimum conditions were $18.8 \%$ wt of polymer concentration, $23 \mathrm{kV}, 11.67 \mu \mathrm{L} / \min$ and $10 \mathrm{~cm}$ in distance.

This study was provided by the Thammasat University scholarship and National Research Council of Thailand. Scanning Electron Microscope was supported by TU-ARC.

\section{References}

1. B. Dhandayuthapani, U.M. Krishnan, S. Sethuraman, J. Biomed Mater Res B Appl Biomater, 94 (2010)

2. R. Jalili, A. Hosseini, M. Morshed, Iran Polym J, 14 (2005)

3. R.Y. Tsai, S.C. Hung, J.Y. Lai, D.M.Wang , H.J. Hsieh, J Taiwan Inst Chem E, 45 (2014)

4. J. Anu Bhushani, C. Anandharamkrishnan, Trends Food Sci Technol 38, (2014)

5. A. Rogina, Appl. Surf. Sci. 296 (2014)

6. A. Baji, Y.W. Mai, S.C. Wong, M. Abtahi, P. Chen, Compos. Sci. Technol. 70 (2010)

7. A.G. Sener, A.S. Altay, F. Altay, 7th IEEE, pp. I-324I-328M. (2011)

8. S. Zargham, S. Bazgir, A. Tavakoli, A.S. Rashidi, R. Damerchely, J. Eng. Fiber. Fabr. 7, $42-49$ (2012)

9. R. Erdem, I. Usta, M. Akalin, O. Atak, M. Yuksek, A. Pars, Appl. Surf. Sci. 334, 227-230 (2014)

10. C. Santos, C.J. Silva, Z. Büttel, R. Guimarães, S.B. Pereira, P. Tamagnini, A. Zille, Carbohydr Polym. 99, 584-592 (2014)

11. Z. Li, C. Wang, Springer Briefs in Materials. pp.1528 (2013)

12. S. Haider, W.A. Al-Masry, N. Bukhari, M. Javid, Polym Eng Sci 50, 1887-1893 (2010)

13. S. Khalili, S. Khorasani, N. Saadatkish, K. Khoshakhlagh, Polym Sci 58, 399-408 (2016)

14. E. Vatankhah, M.P. Prabhakaran, G. Jin, L.G. Mobarakeh, S. Ramakrishna, J Biomater Appl. 28( 6), 909-921 (2014)

15. D. Hussain, F. Loyal, A. Greiner, J.H. Wendorff, Polym J. 51, 3989-3997 (2010)

16. M.A. Oraby, A.I. Waley, A.I El-Dewany, E.A. Saad, B.M. Abd El-Hady, J Appl Sci Res 9, 534-540. (2013). 17. S.O.Han, J.H. Youk, K.D. Min, Y.K. Kang, W.H. Park, Mater Lett 62, 759-762. (2008)

18. M. Chowdhury, G. Stylios, Int J Basic Appl Sci 10 (2010)

19. X. Yuan, Y. Zhang, C. Dong, J. Sheng, Polym Int 53, 1704-1710. (2004)

20. D. Rodoplu, M. Mutlu, J Eng Fiber Fabr 7, 118-123. (2012) 